\title{
Incidence and predictors of syncope recurrence after cardiac pacing in patients with carotid sinus syndrome ir
}

\author{
Giulia Rivasi a , Diana Solari ${ }^{\text {b }}$, Martina Rafanelli ${ }^{\text {a }}$, Alice Ceccofiglio ${ }^{\text {a }}$, Francesca Tesi ${ }^{\text {a }}$, Richard Sutton ${ }^{\text {c }}$, \\ Michele Brignole ${ }^{\mathrm{b}}$, Andrea Ungar ${ }^{\mathrm{a}, *}$
}

a Syncope Unit, Geriatric Intensive Care Medicine, University of Florence and Azienda Ospedaliero Universitaria Careggi, Florence, Italy
b Arrhythmology Centre, Department of Cardiology, Ospedali del Tigullio, Lavagna, Italy

${ }^{c}$ National Heart \&' Lung Institute, Imperial College, London, United Kingdom

\section{A R T I C L E I N F O}

\section{Article history:}

Received 26 January 2018

Received in revised form 13 March 2018

Accepted 31 March 2018

\section{Keywords:}

Carotid sinus syndrome

Cardiac pacing

Carotid sinus massage

Pacemaker

\begin{abstract}
A B S T R A C T
Background: Cardiac pacing is the treatment of choice for cardioinhibitory carotid sinus syndrome (CSS), but syncope recurrence occurs in up to $20 \%$ of patients within 3 years. The present study aims at assessing incidence and identifying predictors of syncope recurrence in patients receiving pacing therapy for CSS.

Methods: The Syncope Clinics of two large regional hospitals in Northern Italy, both following European Syncope Guidelines, combined to perform this study. Retrospective analysis of 3127 consecutive patients undergoing carotid sinus massage (CSM) was performed 2004-2014. Ten-second supine and standing CSM was systematically assessed in patients aged $>40$ years with suspected reflex syncope as part of the initial evaluation. Syncope recurrence was investigated in those paced for CSS having $>6$ months' available follow-up. Data were collected from clinical records and patient interviews.

Results: CSS was diagnosed in 261 patients (8.3\%). Pacemakers were implanted in 158, with follow-up data available in 112: 19 (17\%) experienced 73 syncope recurrences during a mean follow-up of $89 \pm 42$ months, yielding an incidence of 0.5 episodes per patient/year. Prodrome, predisposing situations preceding syncope and chronic nitrate therapy were more frequent in patients reporting recurrence. Prodrome and predisposing situations remained independent predictors of post-implantation recurrence on multivariable analysis.

Conclusions: CSS is a frequent cause of syncope, if CSM is performed during the initial evaluation. Most patients treated by pacing remain asymptomatic during long-term follow-up. In those who have recurrence, its incidence is very low. Prodrome and predisposing situations are predictors of post-implantation recurrence, suggesting presence of hypotensive susceptibility.
\end{abstract}

(C) 2018 Elsevier B.V. All rights reserved.

\section{Introduction}

Carotid sinus syndrome (CCS) is defined as reproduction of spontaneous syncope by means of carotid sinus massage (CSM) associated with asystole $>3 \mathrm{~s}$ and/or a fall in systolic blood pressure (BP) of $>50 \mathrm{~mm} \mathrm{Hg}$ or more [1]. CSS is different from carotid sinus hypersensitivity (CSH) in which asystole or BP fall are demonstrated on CSM but the patient is asymptomatic. A highly variable prevalence of CSS is reported in the literature, ranging from $0 \%$ up to $40 \%$ [2], due to different patient selection, timing of CSM or failure to perform CSM during the work-up.

\footnotetext{
is All authors take responsibility for all aspects of the reliability and freedom from bias of the data presented and their discussed interpretation.

* Corresponding author at: Syncope Unit, Geriatric Intensive Care Medicine, University of Florence and Azienda Ospedaliero-Universitaria Careggi, Viale Pieraccini 6, 50139 Florence, Italy.

E-mail address: aungar@unifi.it (A. Ungar).
}

Cardiac pacing is the treatment of choice for cardioinhibitory (CI) and mixed forms and is a Class IIA recommendation in the European Society of Cardiology (ESC) syncope guidelines [1]; nevertheless, there is still controversy as to the efficacy of this treatment. The evidence supporting this recommendation is considered to be weak, with only four randomized controlled trials [3-6] and a single randomized, double-blind, placebo-controlled trial [7] ever reported, presenting conflicting results. Moreover, syncope recurrence is expected to occur in up to $20 \%$ of patients after pacemaker (PM) implantation [8]. According to the available literature, mixed CSS and a positive response to Tilt Testing (TT) are associated with a higher risk of recurrence $[9,10]$, suggesting a hypotensive underlying mechanism.

The aim of the present study was to assess incidence and identify predictors of syncope recurrence in patients receiving pacing therapy for CSS when CSM was performed in patients aged $>40$ years with suspected reflex syncope as part of the initial evaluation, as recommended by the ESC guidelines on syncope [1]. Two large regional 
hospitals in Northern Italy participated, both following ESC guidelines collected the patients who had been referred for investigation of syncope.

\section{Methods}

The study population consisted of patients who had received cardiac pacing therapy because of mixed or cardioinhibitory CSS. We retrieved electronic records concerning 3127 consecutive patients who had undergone CSM in the Syncope Units of Careggi Hospital, Florence, and Ospedali del Tigullio, Lavagna, Italy in the period 2004-2014. Patients were referred to the Syncope Unit from the Emergency Department, as inpatients or from out-of-hospital services, because of syncope, pre-syncope or unexplained falls. All patients were evaluated according to the ESC guidelines protocol [1] and CSM was performed in patients aged $>40$ years with suspected reflex syncope after initial evaluation (which consisted of clinical history, physical examination, standard 12 lead electrocardiogram, blood pressure measurement in supine and upright positions). Clinical history was aimed at assessing characteristics of spontaneous episodes, including presence of vasovagal prodrome (blurring/clouded vision, light-headedness, loss of balance, pallor, sweating, etc.) and the following predisposing situations for reflex syncope: hot and crowded environment, emotional distress (including intense pain, blood and instrumentation), prolonged standing, typical trigger for situational syncope (micturition, defaecation, cough, post-exercise and post-prandial).

CSM was performed according to the ESC guidelines technique [1]: longitudinal massage was applied for $10 \mathrm{~s}$ over the point of maximum carotid impulse (between the angle of the jaw and the cricoid cartilage, on the anterior margin of the sternocleidomastoid muscle), on the right and then left side. Patients had supine and then erect CSM, using a motorized footplate tilt table with an angle of $60^{\circ}$. The time interval between massages had to be long enough for baseline heart rate (HR) and BP to be restored. In accordance with the "Method of Symptoms" [11], CSH was diagnosed if CSM elicited abnormal cardioinhibition (asystole $\geq 3 \mathrm{~s}$ ) and/or vasodepression (systolic BP fall $\geq 50 \mathrm{~mm} \mathrm{Hg}$ ); if spontaneous symptoms were reproduced in the presence of CSH, CSS was diagnosed. Asymptomatic CSH was not considered diagnostic owing to its low specificity [12]. A transient ischaemic attack, stroke or a myocardial infarction over the previous three months was a contraindication to CSM. In case of carotid bruit, patients were referred for a Doppler ultrasound; if a carotid stenosis $>70 \%$ was detected, CSM was not performed. In order to investigate the susceptibility to orthostatic stress, the evaluation was completed by means of TT performed according to the Italian protocol [13]; positive responses were defined according to the VASIS classification (Vasovagal Syncope International Study) [14]. CSM and TT were performed under continuous electrocardiogram and BP monitoring (Task Force ${ }^{\circledR}$ monitor, CNSystems Medizintechnik AG, Graz, Austria). Written informed consent for the procedures was obtained from each participant.

\subsection{Treatment and follow-up}

Enrolled patients had received a dual-chamber pacemaker. Outcome of the study was recurrence of syncope after pacemaker implantation. Syncopal recurrence was investigated by retrieving clinical records and patient interviews, by telephone or during clinic visits.

\subsection{Statistical analysis}

Data are reported as a mean \pm standard deviation, median with interquartile range or as percentage, as appropriate. The Fisher exact text was used to compare dichotomous variables; the Student $t$-test for unpaired data was used to compare continuous data with normal distribution; the non-parametric Mann-Whitney test for unpaired data was used to compare continuous data with not normal distribution. A multivariable analysis by Cox logistic regression of predictors of syncope recurrence was performed among those variables listed in Table 1 that had a $P$ value $\leq 0.1$ at univariable analysis. The hazard

Table 1

Comparison between paced patients with and without syncope recurrence during a $3.8 \pm 3.4$ year follow-up (min $>6$ months).

\begin{tabular}{|c|c|c|c|c|}
\hline & $\begin{array}{l}\text { All patients } \\
(\mathrm{n}=112)\end{array}$ & $\begin{array}{l}\text { Recurrence } \\
(\mathrm{n}=19)\end{array}$ & $\begin{array}{l}\text { No recurrence } \\
(n=93)\end{array}$ & $P$ \\
\hline Mean follow-up, months \pm SD & $71 \pm 43$ & $89 \pm 42$ & $68 \pm 43$ & 0.06 \\
\hline Mean age, years $\pm S D$ & $77.1 \pm 9.7$ & $78.3 \pm 6.5$ & $76.9 \pm 10.2$ & 0.6 \\
\hline Male sex, n (\%) & $77(69)$ & $10(53)$ & $67(72)$ & 0.09 \\
\hline Number of syncope before PM, median (IQR) & $2(1 ; 3)$ & $3(1 ; 3.5)$ & $2(1 ; 3)$ & 0.3 \\
\hline Number of syncope episodes in the 2 years before evaluation, median (IQR) & $2(1 ; 2)$ & $2(1 ; 3)$ & $1(1 ; 2)$ & 0.5 \\
\hline Incidence of syncope episodes in the 2 years before evaluation, $n / y e a r$ & 0.91/year & 1.03/year & $0.88 /$ year & \\
\hline History of syncope (years), median (IQR) & $1(0.5 ; 4)$ & $1(1 ; 5.5)$ & $1(0.5 ; 4)$ & 0.9 \\
\hline Hypertension, $\mathrm{n}(\%)$ & $52(46.4)$ & $9(47.4)$ & $43(46.2)$ & 0.9 \\
\hline Diabetes, n (\%) & $23(20.5)$ & $3(15.8)$ & $20(21.5)$ & 0.6 \\
\hline Falls, $\mathrm{n}(\%)$ & $21(18.8)$ & $4(21.1)$ & $17(18.9)$ & 0.8 \\
\hline Orthostatic hypotension, n (\%) & $35(31.2)$ & $5(26.3)$ & $30(32.3)$ & 0.6 \\
\hline Heart disease, $\mathrm{n}(\%)$ & $37(33.0)$ & $7(36.8)$ & $30(32.3)$ & 0.7 \\
\hline Presyncope, n (\%) & $45(40.2)$ & $7(36.8)$ & $38(40.9)$ & 0.7 \\
\hline Prodrome, n (\%) & $72(64.3)$ & $16(84.2)$ & $56(60.2)$ & 0.04 \\
\hline Prodrome $>10$ s, n (\%) & $29(25.9)$ & $9(47.4)$ & $20(21.5)$ & 0.02 \\
\hline Prodrome $<10$ s, n (\%) & $43(38.4)$ & $7(36.8)$ & $36(38.7)$ & 0.9 \\
\hline Predisposing situations for VVS, n (\%) & $20(17.9)$ & $6(31.6)$ & $14(15.1)$ & 0.08 \\
\hline Hospitalization for syncope, $\mathrm{n}(\%)$ & $29(25.9)$ & $3(15.8)$ & $26(28.0)$ & 0.3 \\
\hline Injuries, n (\%) & $39(34.8)$ & $5(26.3)$ & $34(36.6)$ & 0.4 \\
\hline Digitalis, n (\%) & $6(5.4)$ & $1(5.3)$ & $5(5.4)$ & 0.9 \\
\hline ACEi/ARB, n (\%) & $51(45.5)$ & $11(57.9)$ & $40(43.0)$ & 0.2 \\
\hline$\beta$-Blockers, n (\%) & $24(21.4)$ & $6(31.6)$ & $18(19.4)$ & 0.2 \\
\hline Calcium channel antagonists, n (\%) & $18(16.1)$ & $5(26.3)$ & $13(14.0)$ & 0.2 \\
\hline Alpha-receptor blockers, n (\%) & $18(16.1)$ & $2(10.5)$ & $16(17.2)$ & 0.5 \\
\hline Nitrates, n (\%) & $7(6.3)$ & $3(15.8)$ & $4(4.3)$ & 0.05 \\
\hline Diuretics, n (\%) & $27(24.1)$ & $6(31.6)$ & $21(22.6)$ & 0.4 \\
\hline Antiarrhythmic drug, n (\%) & $3(2.7)$ & $1(5.3)$ & $2(2.15)$ & 0.4 \\
\hline Other hypotensive drugs, n (\%) & $15(13.4)$ & $3(15.8)$ & $12(12.9)$ & 0.7 \\
\hline Abnormal ECG, n (\%) & $58(51.8)$ & $10(52.6)$ & $48(51.6)$ & 0.9 \\
\hline Atrial fibrillation, $\mathrm{n}(\%)$ & $12(10.7)$ & $1(5.3)$ & $11(11.8)$ & 0.4 \\
\hline Left bundle branch block, n (\%) & $8(7.1)$ & $3(15.8)$ & $5(5.4)$ & 0.1 \\
\hline Right bundle branch block alone, n (\%) & $10(8.9)$ & $1(5.3)$ & $9(9.7)$ & 0.3 \\
\hline Right and anterior fascicular block, $\mathrm{n}(\%)$ & $12(10.7)$ & $1(5.3)$ & $11(11.8)$ & 0.7 \\
\hline I degree atrio-ventricular block, n (\%) & $18(16.1)$ & $1(5.3)$ & $17(18.3)$ & 0.2 \\
\hline Cardioinhibitory CSS, n (\%) & $81(72.3)$ & $14(73.7)$ & $67(72.0)$ & 0.8 \\
\hline Mixed CSS, n (\%) & $31(27.8)$ & $5(26.3)$ & $26(28.0)$ & 0.8 \\
\hline Asystole duration, seconds \pm SD & $7.0 \pm 2.5$ & $6.8 \pm 1.6$ & $7.1 \pm 2.7$ & 0.9 \\
\hline Tilt table test: performed, pts. n (\%) & $89(79 \%)$ & 17 (89\%) & $72(77 \%)$ & 0.3 \\
\hline - of whom positive responses, pts. n (\%) & $44(49.4)$ & $9(53)$ & $35(48.6)$ & 0.5 \\
\hline - VASIS I, n (\%) & $19(21.3)$ & $4(23.5)$ & $15(20.8)$ & 0.6 \\
\hline - VASIS II (A + B), n (\%) & $8(8.9)$ & $0(0.0)$ & $8(11.1)$ & 0.11 \\
\hline - VASIS III, n (\%) & $17(19.1)$ & $5(29.4)$ & $12(16.6)$ & 0.3 \\
\hline
\end{tabular}

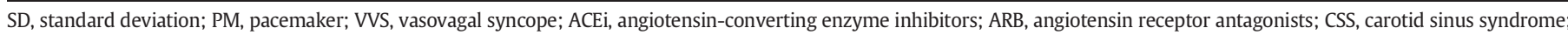
ECG, electrocardiogram. 
ratio (HR) was provided with its $95 \%$ confidence interval (CI). $P<0.05$ was considered to be statistically significant. Analyses were performed using Statistica version 8.0 (StatSoft Italia, Padova, Italy).

\section{Results}

Of a total of 3127 consecutive patients who underwent CSM for suspected reflex syncope after initial evaluation (1272 patients evaluated in Florence and 1855 in Lavagna), CSS was diagnosed in 261 patients (8.3\%): 141 (54\%) had CI, 88 (33.7\%) had mixed and 32 (12\%) had vasodepressor forms. A pacemaker was implanted in $158(69 \%)$ of those with $\mathrm{CI}$ or mixed form who potentially had an indication for pacing; these patients form the study group. In the remaining patients, reasons for not implanting a pacemaker were physician's decision or refusal by the patient. Their clinical characteristics are compared in Supplementary Table 1 . Moreover, 46 patients were lost to follow-up before 6 months, thus finally 112 paced patients in whom follow-up of $\geq 6$ months was available were included for analysis (Fig. 1). The baseline clinical features of the study population are listed in Table 1 . The study participants and patients lost to follow-up had comparable characteristics, with the exception of prolonged prodrome and right bundle branch block, which had a higher prevalence in the study population;
Table 2

Multivariable Cox logistic regression analysis: predictors of recurrence.

\begin{tabular}{lll}
\hline Variables & HR $(95.0 \% \mathrm{CI})$ & $P$ \\
\hline Prodrome & $5.10(1.12-23.29)$ & 0.04 \\
Predisposing situations & $3.76(1.07-13.16)$ & 0.04 \\
Chronic nitrate therapy & $6.17(0.91-41.8)$ & 0.06 \\
Females & $2.56(0.79-8.33)$ & 0.12 \\
Duration of follow-up & $1.01(0.99-1.02)$ & 0.17 \\
\hline
\end{tabular}

the median number of syncope episodes before PM implantation was higher in patients lost to follow-up (Supplementary Table 1).

During a mean follow-up of $89 \pm 42$ months, a total of 73 episodes of syncope recurred in $19 / 112$ patients (17\%) with a median of 2 (IQR 2; 4.5) episodes per patient, yielding an incidence of 0.5 episodes per patient/year of follow-up. At univariable analysis, patients with syncope recurrence more frequently had prodrome, often prolonged, predisposing situations preceding syncope and chronic nitrate therapy. On the contrary, neither the type of response during CSM (CI versus mixed) nor a positive response during TT were able to identify patients with syncope recurrence. At the multivariate Cox logistic regression model, prodrome and predisposing situations remained independent predictors of syncope recurrence (Table 2).

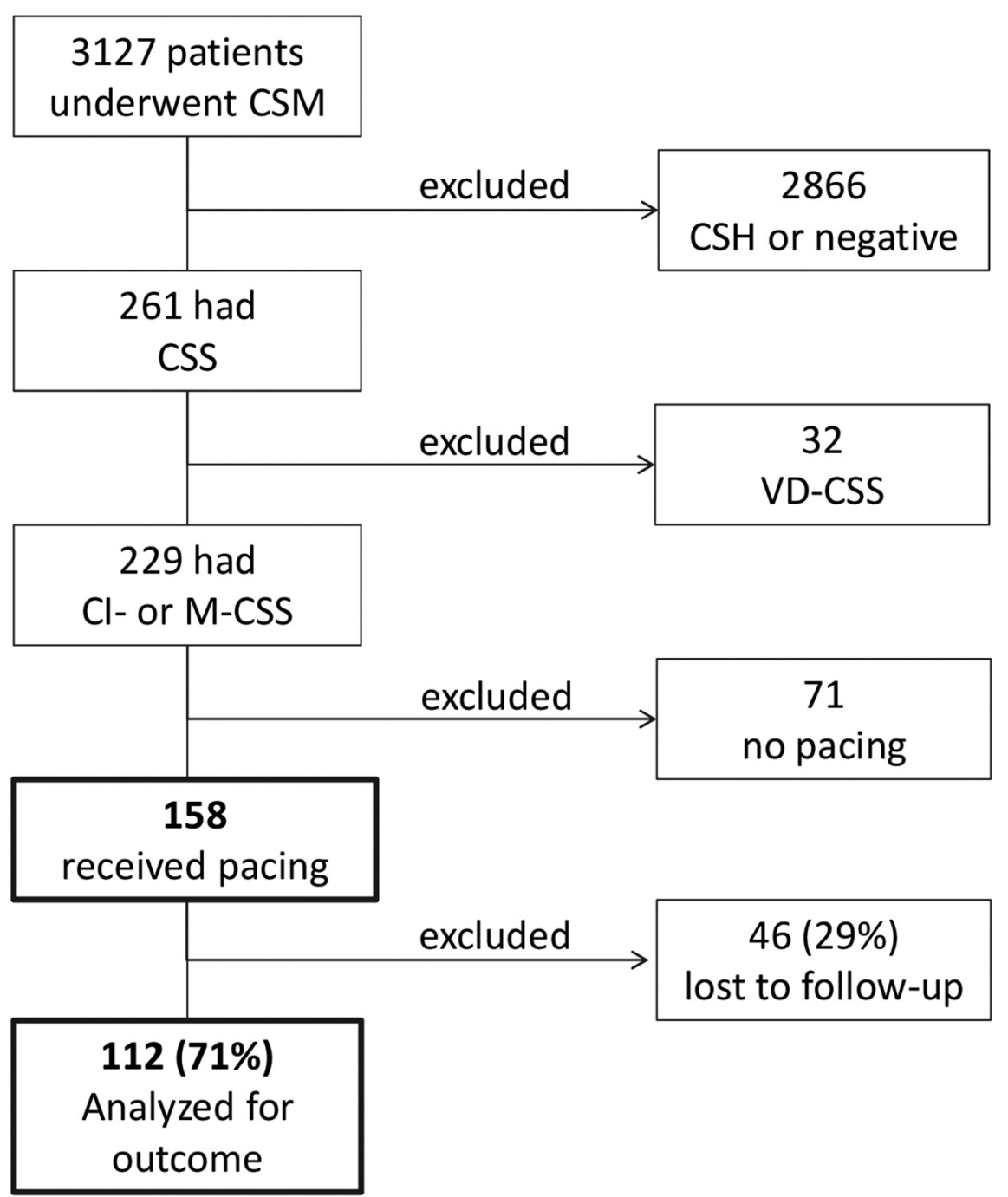

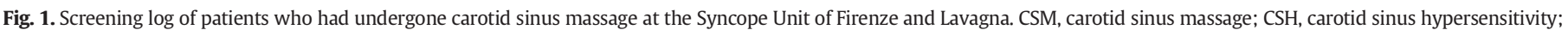
CI-CSS, cardio-inhibitory carotid sinus syndrome; M-CSS, mixed CSS; VD-CSS, vasodepressor CSS. 


\section{Discussion}

Syncope recurrence after cardiac pacing occurred in $17 \%$ of patients having CSS, consistent with previous studies $[15,16]$. We showed that both prodrome and predisposing situations for reflex syncope are associated with a higher risk of syncope recurrence after pacing therapy for mixed or CI-CSS. These predictors can be easily assessed from medical history, allowing the clinician to identify patients at higher risk of recurrence, before cardiac pacing is performed.

The present study validated the indication for CSM given by the ESC guidelines on syncope [1]. In accordance with those guidelines, CSM was systematically performed after initial evaluation in patients aged $>40$ years with suspected reflex syncope, after cardiac aetiology had been excluded. In this clinical context, CSS was diagnosed in $8.3 \%$ of patients and $60.5 \%$ of them received pacing therapy. With the above indications, CSM may increase the diagnostic yield of initial evaluation, guide patients' therapeutic management and avoid execution of other more complex tests.

Performing CSM in accordance with these indications and the restrictive diagnostic criteria of the "Method of Symptoms", cardiac pacing was fairly effective in this large population during a long follow-up: syncope never recurred in the majority of patients and, in the minority who had some recurrence, the incidence fell 10-times from 1.03/year before pacemaker implantation to 0.1 /year after the implantation. Admittedly, not being a controlled trial, we are not able to ascertain the relative contribution of other factors to the decrease in recurrence rate. It is a common finding that syncopal recurrences often decrease spontaneously after medical assessment [17], even in the absence of a specific therapy; in general syncope recurs in $<50 \%$ of patients within 1-2 years and, if recurrence occurs, the burden of syncope decreases even $>70 \%$ compared with the preceding period. In a study by Solari et al. [9] evaluating patients affected by CSS, the yearly syncope burden decreased by a similar magnitude in patients treated with a pacemaker as well as in patients treated with discontinuation/reduction of hypotensive therapies and in those left untreated. Several potential clinical, statistical and psychological explanations have been provided and each of them probably plays a role: patient education and reassurance, an expectation effect or a regression-to-the-mean effect [18].

The mechanism of syncope recurrence after pacemaker implantation may be unclear. PM malfunction is reported to be very rare in these patients [19]. Contrary to other studies in the literature, we were unable to show an increased rate of mixed CSS $[10,19]$ and positive TT $[9,15,20]$ in our patients who had recurrence; the reason is unclear. Nevertheless, some hypotensive susceptibility, which is frequent in patients with reflex syncope, is likely to be involved [21]. The presence of prodrome and/or predisposing situations for reflex syncope suggests that a pronounced hypotensive susceptibility is present, so caution is recommended before referring the patient for pacing therapy. Indeed, hypotensive susceptibility is uninfluenced by pacing and requires specific treatment. Therefore, each patient should receive lifestyle counselling concerning early recognition of prodromal symptoms, prevention of typical predisposing situations and performance of counter-pressure manoeuvres. In addition, reduction or withdrawal of hypotensive therapy should be considered, particularly in cases of polypharmacy; indeed, vasoactive drugs may exacerbate hypotensive susceptibility, particularly in older patients, due to an age-related reduction in peripheral alpha-adrenergic response to orthostatism. In our study population, chronic nitrate therapy was more common in patients with syncope recurrence. Reducing or discontinuing hypotensive therapy may help prevent syncope recurrence, particularly in older patients, as a recent study confirms [22].

\subsection{Limitations}

This is a retrospective study in which no diaries of symptoms were collected by patients, so a wrong or inaccurate recall cannot be excluded; in addition, the precise date and time to syncope recurrence could not be evaluated, thus we were unable to build survival analysis curves. During follow-up, the effects of hypotensive therapy reduction or withdrawal were not investigated, as well as presyncope or minor symptom recurrence.

All the patients were followed periodically in the pacemaker clinic. No data concerning alternative diagnoses was reported. Of course, we cannot exclude that a more accurate reappraisal would have been able to determine associated conditions which could have been responsible for recurrence of syncope. In our study population TT was not able to predict post-implantation recurrence. This result could be related to a reduced sample size: indeed, TT was performed in 17 of 19 patients with recurrence and only 9 positive responses were observed.

Unfortunately, a relevant percentage of paced patients was lost to follow-up, probably due to the retrospective design of our study and the prolonged enrollment period. Not all the patients with a diagnosis of CI or mixed CSS made at initial evaluation finally received a pacemaker therapy. Owing to its retrospective design we can only speculate on the patient's or physician's reasons for not implanting a pacemaker in about one third of them (Fig. 1). They differ for several baseline clinical baseline characteristics from those who implanted a pacemaker (see Supplementary Table 1).

\section{Conclusions}

In case of suspected reflex syncope after the initial evaluation, carotid sinus massage provides a diagnosis of carotid sinus syndrome in $8.3 \%$ of patients; the majority of those affected by $\mathrm{CI}$ or mixed form received pacing therapy (69\%). Cardiac pacing is the treatment of choice for cardioinhibitory carotid sinus syndrome, but syncope recurrence may occur. Prodrome and spontaneous episodes occurring in typical predisposing situations for reflex syncope are predictive of postimplantation recurrence but not a positive tilt test. These predictors can easily be assessed from medical history and are suggestive of hypotensive susceptibility, which should receive specific treatment.

Supplementary data to this article can be found online at https://doi. org/10.1016/j.ijcard.2018.03.144.

\section{Funding}

None.

\section{Conflicts of interest}

Dr. Sutton reports grants and personal fees from acting as Consultant to Medtronic Inc., personal fees from membership of Speakers' Bureau SJM Abbott, other as Stockholder in Boston Scientific, Edwards Lifesciences, Shire, Roche, AstraZeneca, outside the submitted work. All other authors have nothing to disclose.

\section{References}

[1] A. Moya, R. Sutton, F. Ammirati, et al., Guidelines for the diagnosis and management of syncope (version 2009): the Task Force for the Diagnosis and Management of Syncope of the European Society of Cardiology (ESC), Eur. Heart J. 30 (2009) 2631-2671.

[2] R. Sutton, Carotid sinus syndrome: progress in understanding and management, Glob. Cardiol. Sci. Pract. 2014 (2014) 1-8.

[3] M. Brignole, C. Menozzi, G. Lolli, B. Sartore, M. Barra, Natural and unnatural history of patients with severe carotid sinus hypersensitivity: a preliminary study, Pacing Clin. Electrophysiol. 11 (1988) 1628-1635.

[4] R.A. Kenny, D.A. Richardson, N. Steen, R.S. Bexton, F.E. Shaw, J. Bond, Carotid sinus syndrome: a modifiable risk factor for non-accidental falls in older adults (SAFE PACE), J. Am. Coll. Cardiol. 38 (2001) 1491-1496.

[5] J.E. Claesson, B.O. Kristensson, N. Edvardsson, P. Währborg, Less syncope and milder symptoms in patients treated with pacing for induced cardioinhibitory carotid sinus syndrome: a randomized study, Europace 9 (2007) 932-936.

[6] D.J. Ryan, S. Nick, S.M. Colette, R.A. Kenny, Carotid sinus syndrome, should we pace? A multicentre, randomized controlled trial (SAFE PACE 2), Heart 96 (2010) 347-351. 
[7] S.W. Parry, N. Steen, R.S. Bexton, M. Tynan, R.A. Kenny, Pacing in elderly recurrent fallers with carotid sinus hypersensitivity: a randomized, double-blind, placebo controlled crossover trial, Heart 95 (2009) 405-409.

[8] M. Brignole, Pacing for neurally-mediated syncope: how to decide? Cardiol. J. 21 (2014) 601-605.

[9] D. Solari, R. Maggi, D. Oddone, et al., Clinical context and outcome of carotid sinus syndrome diagnosed by means of the 'method of symptoms', Europace 16 (2014) 928-934.

[10] R. Lopes, A. Gonçalves, J. Campos, et al., The role of pacemaker in hypersensitive carotid sinus syndrome, Europace 13 (2011) 572-575.

[11] E. Puggioni, V. Guiducci, M. Brignole, et al., Results and complications of the carotic sinus massage performed according to the "methods of symptoms", Am. J. Cardiol. 89 (2002) 599-601.

[12] C.T. Krediet, S.W. Parry, D.L. Jardine, D.G. Benditt, M. Brignole, W. Wieling, The history of diagnosing carotid sinus hypersensitivity: why are the current criteria too sensitive? Europace 13 (2011) 14-22.

[13] A. Bartoletti, P. Alboni, F. Ammirati, et al., "The Italian Protocol": a simplified head-up tilt testing potentiated with oral nitroglycerin to assess patients with unexplained syncope, Europace 2 (2000) 339-342.

[14] R. Sutton, M. Petersen, M. Brignole, A. Raviele, C. Menozzi, P. Giani, Proposed classification for tilt induced vasovagal syncope, Eur. J. Cardiac Pacing Electrophysiol. 3 (1992) 180-183.

[15] G. Gaggioli, M. Brignole, C. Menozzi, et al., A positive response to head-up tilt testing predicts syncopal recurrence in carotid sinus syndrome patients with permanent pacemakers, Am. J. Cardiol. 76 (1995) 720-722.
[16] M. Brignole, F. Ammirati, F. Arabia, et al., Syncope Unit Project (SUP) Two Investigators, Assessment of a standardized algorithm for cardiac pacing in older patients affected by severe unpredictable reflex syncope, Eur. Heart J. 36 (2015) 1529-1535.

[17] P. Pournazari, I. Sahota, R. Sheldon, High remission rates in vasovagal syncope: systematic review and meta-analysis of observational and randomized studies, JACC: Clin. Electrophysiol. 3 (4) (2017) 384-392.

[18] IS. Sahota, C Maxey, P. Pournazari, R. Sheldon, Clusters, gaps, and randomness: vasovagal syncope recurrence patterns, JACC: Clin. Electrophysiol. 3 (9) (2017) 1046-1053.

[19] R. Sutton, Syncope in patients with pacemakers, Arrhythmia Electrophysiol. Rev. 4 (2015) 189-192.

[20] M. Brignole, P. Donateo, M. Tomaino, et al., Benefit of pacemaker therapy in patients with presumed neurally mediated syncope and documented asystole is greater when tilt test is negative: an analysis from the third International Study on Syncope of Uncertain Etiology (ISSUE-3), Circ. Arrhythm. Electrophysiol. 7 (2014) 10-16.

[21] R. Sutton, M. Brignole, Twenty-eight years of research permit reinterpretation of tilttesting: hypotensive susceptibility rather than diagnosis, Eur. Heart J. 35 (2014) 2211-2212.

[22] D. Solari, F. Tesi, M. Unterhuber, et al., Stop vasodepressor drugs in reflex syncope: a randomised controlled trial, Heart 103 (2017) 449-455. 